\title{
Micromechanical effective elastic moduli of continuous fiber-reinforced composites with near-field fiber interactions
}

Received: 28 March 2010 / Published online: 30 June 2010

(C) The Author(s) 2010. This article is published with open access at Springerlink.com

\begin{abstract}
A higher-order micromechanical framework is presented to predict the overall elastic deformation behavior of continuous fiber-reinforced composites with high-volume fractions and random-fiber distributions. By taking advantage of the probabilistic pair-wise near-field interaction solution, the interacting eigenstrain is analytically derived. Subsequently, by making use of the Eshelby equivalence principle, the perturbed strain within a continuous circular fiber is accounted for. Further, based on the general micromechanical field equations, effective elastic moduli of continuous fiber-reinforced composites are constructed. An advantage of the present framework is that the higher-order effective elastic moduli of composites can be analytically predicted with relative simplicity, requiring only material properties of the matrix and fibers, the fiber-volume fraction and the microstructural parameter $\gamma$. Moreover, no Monte Carlo simulation is needed for the proposed methodology. A series of comparisons between the analytical predictions and the available experimental data for isotropic and anisotropic fiber reinforced composites illustrate the predictive capability of the proposed framework.
\end{abstract}

\section{Introduction}

The requirement for higher structural efficiency (a combination of stiffness and strength normalized by density) provides a significant motivation for the development of improved materials for a multitude of engineering applications. Correspondingly, composite materials have been widely studied and employed in diverse fields of science and engineering disciplines. In comparison with many conventional materials (such as steel and aluminum), the particle- or fiber-reinforced composites offer salient features such as low density, high strength-to-weight ratio, high toughness, high stiffness, enhanced creep resistance, better wear resistance, superior environmental durability, custom microstructural morphology, preferred directionality, and so on. The inclusions encompass uni-directionally aligned, bi-directional, or randomly dispersed fibers or particles in a matrix material. The matrix material may consist of metal, ceramic or polymer. In particular, continuous fiber reinforced composites are attractive as they offer outstanding longitudinal mechanical properties compared with composites reinforced by particulates or whiskers. A thorough investigation and understanding of aligned-fiber reinforced composites is requisite for the development of rational design of more serviceable, laminated or woven composites [1].

J. W. Ju $(\varangle) \cdot$ K. Yanase

Department of Civil and Environmental Engineering, University of California,

Los Angeles, CA 90095-1593, USA

E-mail: juj@ucla.edu

URL: http://www.cee.ucla.edu/faculty/ju.htm

Present address:

K. Yanase

Department of Mechanical Engineering, Fukuoka University, Fukuoka, Japan 
Based on specific requirements, fiber-reinforced composites can be tailored by properly choosing the matrix and fibers, fiber volume fractions, fiber alignment, fiber shapes, etc. Accurate predictions on material properties and behaviors of composites, such as effective elastic moduli, are requisite to meeting specific design requirements and criteria. In practice, due to improved stiffness-to-weight ratio and strengthto-weight ratio, fiber-reinforced composites are often constructed with carbon or graphite fibers. The carbon/graphite fibers are the predominant high-strength, high modulus reinforcements and are widely used for high-performance polymer-matrix composites. These carbon/graphite fiber-reinforced composites have been commonly adopted for aerospace, automobile, civil infrastructure, and many other engineering, sporting goods and consumer applications. In the graphite nanostructure, the carbon atoms are arranged in the form of hexagonal layers with a very dense packing in the layer planes. The high strength bond between carbon atoms in the layer planes results in an extremely high modulus. By contrast, the weak van der Waals-type bond between the neighboring layers results in lower modulus [2]. Therefore, the carbon/graphite fibers are highly anisotropic with the longitudinal stiffness an order of magnitude higher than the transverse stiffness [3].

In engineering practice, the finite element method is routinely used to predict the three-dimensional stress/strain fields within heterogeneous materials. In general, to construct a proper finite element formulation, nine elastic moduli of orthotropic heterogeneous materials are required. However, these anisotropic effective elastic properties can be difficult to acquire. Therefore, it is a common practice to approximate the material properties in order to obtain adequate solutions. On the other hand, emanating from Eshelby's approach [4], analytical micromechanical methods are also popular for composite materials, which aim at the theoretical analysis of a representative volume element (RVE) or a representative area element (RAE). A major advantage of the Eshelby-type micromechanical approach and homogenization is that it enables us to predict full multi-axial properties and responses of heterogeneous materials in principle. Based on the pioneering work by Eshelby, the effective medium theories (the Eshelby method, the Mori-Tanaka method, the self-consistent method, the generalized self-consistent method, the differential scheme, etc.) are broadly employed to estimate the effective properties of heterogeneous materials (cf. [5]). However, these effective medium theories are based on the single-inclusion problem, and only the average effects of all other inclusions are considered. In other words, the actual locations, direct inclusion interactions and random dispersions of inclusions are not considered in the effective medium theories. In essence, the direct or near-field inclusion interactions need to be adequately accommodated to predict the effective elastic moduli and deformation responses of heterogeneous composites with random microstructures and high volume fractions of inclusions.

In the literature, some approaches were proposed to tackle the near-field interaction topics. For instance, Moschovidis and Mura [6] considered the polynomial eigenstrain to account for the effect of pair-wise interactions for stress/strain fields. In reality, it is impossible to find the analytical eigenstrain in the presence of many particle interactions. To overcome this difficulty, Ju and Chen [7-9] developed an approximate yet accurate method to account for the inter-particle interaction effects in two-phase composites. The Ju-Chen higherorder (in terms of particle volume fraction) micromechanical formulation can be applied to the modeling of composites with higher volume fractions. Additionally, Ju and Zhang [10,11] developed the higher-order micromechanical formulation for continuous fiber-reinforced composites. Recently, Lin and Ju [12] extended the $\mathrm{Ju}$-Chen pair-wise interaction formulation to the three-phase composites containing many randomly dispersed isotropic spherical particles.

The main objective of the present work is to predict the effective elastic moduli of continuous circular fiber reinforced composites in the presence of strong near-field pair-wise fiber interactions. The proposed methodology emanates from the micromechanics-based probabilistic fiber interaction solution and the homogenization procedure. By taking advantage of the Eshelby equivalence principle and probabilistic microstructural homogenization, the effective elastic moduli of aligned fiber-reinforced composites are analytically derived, featuring the near-field interacting eigenstrains.

\section{Effective elastic moduli of fibrous composites}

\subsection{Pair-wise fiber interactions}

We consider the two-dimensional interactions among the continuous fibers as exhibited by Fig. 1. In the presence of near-field direct interactions, the interior-point Eshelby tensor $\mathbf{S}$ (cf. $[5,13,14]$ ) is not sufficient to describe the perturbed strain field within fibers. The exterior-point Eshelby tensor $\overline{\mathbf{G}}$ can account for the strain 


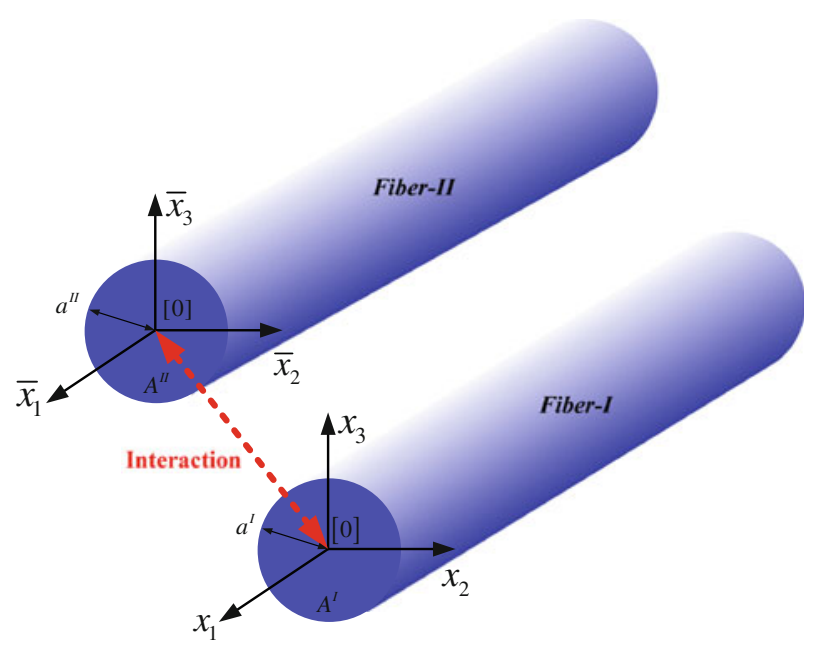

Fig. 1 The two-dimensional pair-wise fiber interaction

field perturbed by the other fiber. Therefore, we have the following equation:

$$
\varepsilon^{I^{\prime}}(\mathbf{x})=\mathbf{S}^{I}: \varepsilon^{I^{* *}}+\overline{\mathbf{G}}^{I I}(\overline{\mathbf{x}}): \varepsilon^{I I^{* *}}
$$

The interior-point Eshelby tensor $\mathbf{S}$ for the prolate sphere $\left(a_{1}>a_{2}=a_{3}\right)$ can be expressed as

$$
\begin{aligned}
8 \pi\left(1-v_{0}\right) S_{i j k l}= & \delta_{i j} \delta_{k l}\left[2 v_{0} I_{I}-I_{K}+a_{I}^{2} I_{K I}\right] \\
& +\left(\delta_{i k} \delta_{j l}+\delta_{j k} \delta_{i l}\right)\left\{a_{I}^{2} I_{I J}-I_{J}+\left(1-v_{0}\right)\left[I_{K}+I_{L}\right]\right\},
\end{aligned}
$$

where

$$
\begin{aligned}
& I_{1}=-\frac{4 \pi a_{3}^{2}}{a_{1}^{2}-a_{3}^{2}}-\frac{2 \pi a_{1} a_{3}^{2}}{\left(a_{1}^{2}-a_{3}^{2}\right)^{3 / 2}}\left\{\ln \frac{a_{1}-\sqrt{a_{1}^{2}-a_{3}^{2}}}{a_{1}+\sqrt{a_{1}^{2}-a_{3}^{2}}}\right\}, \quad I_{2}=I_{3}=\frac{1}{2}\left(4 \pi-I_{1}\right), \\
& I_{12}=I_{21}=I_{13}=I_{31}=\frac{2 \pi}{a_{1}^{2}-a_{3}^{2}}-\frac{3}{2\left(a_{1}^{2}-a_{3}^{2}\right)} I_{1}, \\
& I_{11}=-\frac{4 \pi a_{3}^{2}}{3\left(a_{1}^{2}-a_{3}^{2}\right)} \cdot \frac{1}{a_{1}^{2}}+\frac{I_{1}}{a_{1}^{2}-a_{3}^{2}}, \quad I_{22}=I_{23}=I_{32}=I_{33}=\frac{\pi}{a_{3}^{2}}-\frac{1}{4} I_{12} .
\end{aligned}
$$

Here, $v_{0}$ signifies the Poisson's ratio of the matrix material. Accordingly, by setting $a_{1} / a_{3} \rightarrow \infty$, Eq. (2) can easily reproduce $\mathbf{S}$ for the continuous circular fiber. Furthermore, it is noted that $\overline{\mathbf{G}}(\overline{\mathbf{x}})$ represents the exterior-point Eshelby tensor (cf. [13]) for the continuous circular fiber expressed with the $\overline{\mathbf{x}}$ coordinate system (cf. Fig. 1). The components of $\overline{\mathbf{G}}$ are as follows (cf. $[10,13])$ :

$$
\begin{aligned}
\overline{\mathbf{G}}(r)= & \frac{\rho^{2}}{8\left(1-v_{0}\right)}\left[\left(2-4 v_{0}\right)\left(\delta_{i k} \delta_{j l}+\delta_{i l} \delta_{j k}\right)+\left(4 v_{0}-2\right) \delta_{i j} \delta_{k l}\right. \\
& +4 \delta_{i j} n_{k} n_{l}+4\left(1-2 v_{0}\right) \delta_{k l} n_{i} n_{j}-16 n_{i} n_{j} n_{k} n_{l} \\
& \left.+4 v_{0}\left(\delta_{i k} n_{j} n_{l}+\delta_{i l} n_{j} n_{k}+\delta_{j k} n_{i} n_{l}+\delta_{j l} n_{i} n_{k}\right)\right], \quad i, j, k, l=2,3,
\end{aligned}
$$

where $\rho=a / r, a$ is the fiber radius and $r$ represents the center-to-center distance of fibers, and $\delta_{i j}$ signifies the Kronecker delta. Further, using the polar coordinate system, the components of the unit outward normal vector $\mathbf{n}$ can be rendered as

$$
n_{2}=\cos \theta, \quad n_{3}=\sin \theta
$$

In an advanced composite system, residual stresses commonly exist inside the material. For example, due to the high-temperature manufacturing process, the presence of thermal residual stresses is a common phenomenon in metal-matrix composites. Such residual stresses can be effectively accounted for by introducing the thermal eigenstrain [15-18]. Accordingly, in the presence of thermal eigenstrain, the equivalence equation can 
be expressed as:

$$
\mathbf{C}_{0}:\left(\varepsilon^{0}+\varepsilon^{\prime}-\varepsilon^{* *}\right)=\mathbf{C}^{I}:\left(\varepsilon^{0}+\varepsilon^{\prime}-\varepsilon^{*}\right), \quad \text { where } \varepsilon^{\prime}=\mathbf{S}: \varepsilon^{* *} .
$$

Here, $\varepsilon^{*}$ and $\varepsilon^{* *}$ are the thermal eigenstrain and total eigenstrain, respectively. Further, $\mathbf{C}_{0}$ and $\mathbf{C}^{I}$ represent the fourth-order elastic stiffnesses of the matrix and fiber, respectively. We consider the constant or crosssection-averaged total eigenstrain for the sake of simplicity. Therefore, using Eqs. (1) and (8), the following equation can be attained:

$$
\left(\Delta \mathbf{C}^{I} \bullet \mathbf{S}+\mathbf{C}_{0}\right): \varepsilon^{I^{* *}}+\Delta \mathbf{C}^{I} \bullet \overline{\mathbf{G}}^{I I}(\overline{\mathbf{x}}): \varepsilon^{I I^{* *}}=-\Delta \mathbf{C}^{I}: \varepsilon^{0}+\mathbf{C}^{I}: \varepsilon^{*},
$$

where

$$
\Delta \mathbf{C}^{I}=\mathbf{C}^{I}-\mathbf{C}_{0}
$$

Since the solution of Eq. (9) is position-dependent within the fiber, it is mathematically inconvenient for further derivations. Therefore, by applying the area-averaging in the $A^{I}$ domain with a Taylor expansion (cf. [6], [8]) at the origin of $\mathbf{x}$ coordinate (cf. Fig. 1), Eq. (9) can be recast as:

$$
\begin{aligned}
& \left(\Delta \mathbf{C}^{I} \bullet \mathbf{S}+\mathbf{C}_{0}\right): \varepsilon^{I^{* *}} \\
& \quad+\frac{1}{A^{I}} \Delta \mathbf{C}^{I} \bullet\left\{\int_{A^{I}}\left(\overline{\mathbf{G}}^{I I}[0]+\frac{\partial \overline{\mathbf{G}}^{I I}[0]}{\partial \bar{x}_{m}} x_{m}+\frac{1}{2} \frac{\partial \overline{\mathbf{G}}^{I I}[0]}{\partial \bar{x}_{m} \partial \bar{x}_{n}} x_{m} x_{n}+\cdots\right) \mathrm{d} A\right\}: \varepsilon^{I I^{* *}}=-\Delta \mathbf{C}^{I}: \varepsilon^{0}+\mathbf{C}^{I}: \varepsilon^{*} .
\end{aligned}
$$

Hence, we arrive at:

$$
\begin{aligned}
& \left(\Delta \mathbf{C}^{I} \bullet \mathbf{S}+\mathbf{C}_{0}\right): \varepsilon^{I^{* *}} \\
& +\frac{1}{A^{I}} \Delta \mathbf{C}^{I} \bullet\left\{\overline{\mathbf{G}}^{I I}[0] A^{I}+\frac{\partial \overline{\mathbf{G}}^{I I}[0]}{\partial \bar{x}_{m}} \int_{A^{I}} x_{m} \mathrm{~d} A+\frac{1}{2} \frac{\partial \overline{\mathbf{G}}^{I I}[0]}{\partial \bar{x}_{m} \partial \bar{x}_{n}} \int_{A^{I}} x_{m} x_{n} \mathrm{~d} A+\cdots\right\}: \varepsilon^{I I^{* *}}=-\Delta \mathbf{C}^{I}: \varepsilon^{0}+\mathbf{C}^{I}: \varepsilon^{*},
\end{aligned}
$$

where

$$
\int_{A^{I}} x_{m} \mathrm{~d} A=0, \quad \int_{A^{I}} x_{m} x_{n} \mathrm{~d} A=\frac{a_{I}^{4} \pi}{4} \delta_{m n}=\frac{a_{I}^{2}}{4} A^{I} \delta_{m n} .
$$

Here, $a_{I}$ signifies the radius of fiber-I (cf. Fig. 1). By performing the Taylor expansion up to the second-order, the following equation is rendered:

$$
\left(\Delta \mathbf{C}^{I} \bullet \mathbf{S}^{I}+\mathbf{C}_{0}\right): \varepsilon^{I^{* *}}+\Delta \mathbf{C}^{I} \bullet \hat{\mathbf{G}}^{I I}: \varepsilon^{I I^{* *}}=-\Delta \mathbf{C}^{I}: \varepsilon^{0}+\mathbf{C}^{I}: \varepsilon^{*} .
$$

Further, by using the mean fiber radius (i.e., $a^{I}=a^{I I}=a$ ), $\hat{\mathbf{G}}$ takes the form:

$$
\begin{aligned}
\hat{\mathbf{G}}(r)= & \overline{\mathbf{G}}^{I I}[0]+\frac{a^{2} \cdot \delta_{m n}}{8} \frac{\partial \overline{\mathbf{G}}^{I I}[0]}{\partial \bar{x}_{m} \partial \bar{x}_{n}} \\
= & \frac{\rho^{2}}{8\left(1-v_{0}\right)}\left[\left(\rho^{4}+\rho^{2}+2-4 v_{0}\right)\left(\delta_{i k} \delta_{j l}+\delta_{i l} \delta_{j k}\right)+\left(\rho^{4}+\rho^{2}+4 v_{0}-2\right) \delta_{i j} \delta_{k l}\right. \\
& +4\left(1-\rho^{2}-\rho^{4}\right) \delta_{i j} n_{k} n_{l}+4\left(1-2 v_{0}-\rho^{2}-\rho^{4}\right) \delta_{k l} n_{i} n_{j}+8\left(3 \rho^{4}+3 \rho^{2}-2\right) n_{i} n_{j} n_{k} n_{l} \\
& \left.+4\left(v_{0}-\rho^{2}-\rho^{4}\right)\left(\delta_{i k} n_{j} n_{l}+\delta_{i l} n_{j} n_{k}+\delta_{j k} n_{i} n_{l}+\delta_{j l} n_{i} n_{k}\right)\right]+0\left(\rho^{7}\right) .
\end{aligned}
$$




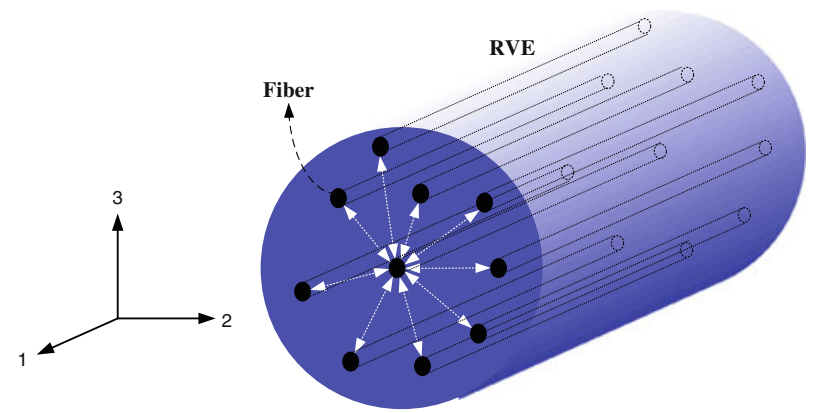

Fig. 2 The probabilistic two-dimensional pair-wise fiber interactions within a cylindrical RVE

In essence, Eq. (15) represents the cross-sectional averaged $\overline{\mathbf{G}}$ within a fiber. By taking advantage of $\hat{\mathbf{G}}$, the intensity of near-field interaction can be accounted for based on the center-to-center distance of fibers in the following derivations.

Based on Eq. (14), the equivalence equations within $A^{I}$ and $A^{I I}$ can be expressed as:

$$
\begin{aligned}
\mathbf{A}^{I}: \varepsilon^{I^{* *}}+\hat{\mathbf{G}}: \varepsilon^{I I^{* *}} & =-\varepsilon^{0}+\mathbf{B}^{I}: \varepsilon^{I^{*}}, \\
\hat{\mathbf{G}}: \varepsilon^{I^{* *}}+\mathbf{A}^{I I}: \varepsilon^{I I^{* *}} & =-\varepsilon^{0}+\mathbf{B}^{I I}: \varepsilon^{I I^{*}},
\end{aligned}
$$

where

$$
\mathbf{A}^{(r)}=\left(\Delta \mathbf{C}^{(r)}\right)^{-1} \bullet \mathbf{C}_{0}+\mathbf{S}, \quad \mathbf{B}^{(r)}=\left(\Delta \mathbf{C}^{(r)}\right)^{-1} \bullet \mathbf{C}^{(r)}
$$

and $(r)=I, I I$. By solving the above equations to find $\varepsilon^{I^{* *}}$, we obtain the following equation:

$$
\left[\mathbf{A}^{I}-\hat{\mathbf{G}} \bullet\left(\mathbf{A}^{I I}\right)^{-1} \bullet \hat{\mathbf{G}}\right]: \varepsilon^{\varepsilon^{* *}}=\left[\hat{\mathbf{G}} \bullet\left(\mathbf{A}^{I I}\right)^{-1}-\mathbf{I}\right]: \varepsilon^{0}+\mathbf{B}^{I}: \varepsilon^{I^{*}}+\hat{\mathbf{G}} \bullet\left(\mathbf{A}^{I I}\right)^{-1} \bullet \mathbf{B}^{I I}: \varepsilon^{I I^{*}}
$$

Here, I defines the fourth-order identity tensor.

\subsection{Pair-wise fiber interaction with conditional probability}

Let us consider the probable location for the second fiber or $\varepsilon^{I I^{* *}}$ (cf. Fig. 2). Therefore, Eq. (19) results in the following equation:

$$
\begin{aligned}
& {\left[\mathbf{A}^{I}-\int_{A \notin A^{I}} \hat{\mathbf{G}} \bullet\left(\mathbf{A}^{I I}\right)^{-1} \bullet \hat{\mathbf{G}} \cdot P\left(\mathbf{x}^{I I} \mid \mathbf{x}^{I}\right) \mathrm{d} A\right]: \varepsilon^{I^{* *}}} \\
& =\left[\int_{A \notin A^{I}} \hat{\mathbf{G}} \bullet\left(\mathbf{A}^{I I}\right)^{-1} \cdot P\left(\mathbf{x}^{I I} \mid \mathbf{x}^{I}\right) \mathrm{d} A-\mathbf{I}\right]: \varepsilon^{0}+\mathbf{B}^{I}: \varepsilon^{I^{*}} \\
& +\left[\int_{A \notin A^{I}} \hat{\mathbf{G}} \bullet\left(\mathbf{A}^{I I}\right)^{-1} \bullet \mathbf{B}^{I I} \cdot P\left(\mathbf{x}^{I I} \mid \mathbf{x}^{I}\right) \mathrm{d} A\right]: \varepsilon^{I I^{*}} .
\end{aligned}
$$

Here, $P\left(\mathbf{x}^{I I} \mid \mathbf{x}^{I}\right)$ signifies the conditional probability density function to find the second fiber in the presence of the first fiber. By integrating Eq. (20) in cylindrical coordinates, we obtain

$$
\left[\mathbf{A}^{I}-\int_{A \notin A^{I}} \hat{\mathbf{G}} \bullet\left(\mathbf{A}^{I I}\right)^{-1} \bullet \hat{\mathbf{G}} \cdot P\left(\mathbf{x}^{I I} \mid \mathbf{x}^{I}\right) \mathrm{d} A\right]: \varepsilon^{I^{* *}}=-\varepsilon^{0}+\mathbf{B}^{I}: \varepsilon^{I^{* *}} .
$$


It is noted that the fourth-order tensors $\overline{\mathbf{G}}$ and $\hat{\mathbf{G}}$ have the following properties:

$$
\begin{aligned}
& \int_{2 a}^{\infty}\left(\int_{0}^{2 \pi} \overline{\mathbf{G}} d \theta\right) r \mathrm{~d} r=0, \\
& \int_{2 a}^{\infty}\left(\int_{0}^{2 \pi} \hat{\mathbf{G}} d \theta\right) r \mathrm{~d} r=0 .
\end{aligned}
$$

The above results can be easily derived by taking advantage of the following relations [10]:

$$
\begin{aligned}
\int_{0}^{2 \pi} n_{i} n_{j} \mathrm{~d} \theta & =\pi \delta_{i j}, \\
\int_{0}^{2 \pi} n_{i} n_{j} n_{k} n_{l} \mathrm{~d} \theta & =\frac{\pi}{4}\left(\delta_{i j} \delta_{k l}+\delta_{i k} \delta_{j l}+\delta_{i l} \delta_{j k}\right) .
\end{aligned}
$$

The result in Eq. (22) can be verified by the Tanaka-Mori lemma [19,20]. In the absence of actual manufacturing evidences, it is often assumed that the two-point conditional probability function is statistically isotropic, uniform, and obeys the following form (cf. [8]):

$$
P\left(\mathbf{x}^{I I} \mid \mathbf{x}^{I}\right)=\left\{\begin{array}{cc}
N^{I I} / \mathrm{A}, & \text { if } r \geq a \\
0, & \text { otherwise }
\end{array}\right.
$$

In the above equation, $\mathrm{A}$ is the area of the RAE, $N / \mathrm{A}$ signifies the number density of fibers, and the following relation holds:

$$
\frac{N^{(r)}}{\mathrm{A}}=\frac{\phi^{(r)}}{\pi a^{2}},
$$

where $\phi^{(r)}$ is the volume fraction of the $r^{\text {th }}$-phase fibers. In essence, Eq. (26) can serve as the simplest approximation since it tends to underestimate the probabilistic existence of a second fiber in the neighborhood of $\mathbf{x}^{I}$ in the event of high fiber-volume fraction. Therefore, Eq. (26) may be regarded as the lower bound for the microstructure. Furthermore, if we assume statistical isotropy, then the two-point conditional probability function depends upon the radial distribution function $g(r)$ :

$$
P\left(\mathbf{x}^{I I} \mid \mathbf{x}^{I}\right)=\frac{N^{I I}}{\mathrm{~A}} g(r) .
$$

As a consequence, Eq. (21) can be recast as:

$$
\left\{\mathbf{A}^{I}-\frac{N^{I I}}{\mathrm{~A}} \int_{A \notin A^{I}} \hat{\mathbf{G}} \bullet\left(\mathbf{A}^{I I}\right)^{-1} \bullet \hat{\mathbf{G}} \cdot g(r) \mathrm{d} A\right\}: \varepsilon^{I^{* *}}=-\varepsilon^{0}+\mathbf{B}^{I}: \varepsilon^{I^{*}} .
$$

Therefore, in the presence of several distinct properties of fibers, the eigenstrain within each phase of fiber can be expressed as

$$
\begin{gathered}
\left(\mathbf{A}^{(1)}-\sum_{r=1}^{N} \overline{\mathbf{A}}^{(r)}\right): \varepsilon^{1^{* *}}=-\varepsilon^{0}+\mathbf{B}^{(1)}: \varepsilon^{1^{*},} \\
\left(\mathbf{A}^{(2)}-\sum_{r=1}^{N} \overline{\mathbf{A}}^{(r)}\right): \varepsilon^{2^{* *}}=-\varepsilon^{0}+\mathbf{B}^{(2)}: \varepsilon^{2^{*}}, \\
\bullet \\
\left(\mathbf{A}^{(N)}-\sum_{r=1}^{N} \overline{\mathbf{A}}^{(r)}\right): \varepsilon^{N^{* *}}=-\varepsilon^{0}+\mathbf{B}^{(N)}: \varepsilon^{N^{*}},
\end{gathered}
$$


where

$$
\overline{\mathbf{A}}^{(r)}=\frac{N^{(r)}}{\mathrm{A}} \int_{A \notin A^{(r)}} \hat{\mathbf{G}} \bullet\left(\mathbf{A}^{(r)}\right)^{-1} \bullet \hat{\mathbf{G}} \cdot g(r) \mathrm{d} A .
$$

After some derivations, the components of the tensor $\overline{\mathbf{A}}^{(r)}$ can be secured (cf. Appendix I).

\subsection{Radial distribution function}

In computational mechanics and statistical mechanics, a radial distribution function (RDF, $g(r)$ ) describes how the density of surrounding matter varies as a function of the distance from a particular point. Under a potential energy function, the radial distribution function can be found via computer simulation such as the Monte Carlo method. It is also possible to use rigorous statistical mechanics to establish a proper RDF. The Percus-Yevick approximation is a well-known solution for the radial distribution function of a hard-sphere liquid. For instance, a tractable form of solution is provided by Trokhymchuk et al. [21] (cf. Appendix II). Since the Percus-Yevick solution is for the three-dimensional case, it tends to overestimate the peak density for the two-dimensional radial distribution. Therefore, a renormalization of fiber-volume fraction is suggested in the literature. Accordingly, by following the approximate method proposed by Everett [22], the normalized fiber-volume fraction $\bar{\phi}^{(r)}$ is adopted to compute RDF:

$$
\bar{\phi}^{(r)}=0.05+0.03 \phi^{(r)}+1.25\left(\phi^{(r)}\right)^{2} .
$$

In Fig. 3, the predicted RDFs with Eq. (32) are compared with the experimental data of uniform-diameter random RDFs for Nicalon fiber/zirconia titanate composites (cf. [22]). Good fits are obtained for each fiber volume fraction up to $\phi=0.5$. Clearly, the assumption of a uniform radial distribution $g(r)=1$ is reasonable only for small fiber volume fractions.

\subsection{Consistent and simplified perturbed strains in continuous circular fibers}

After the interacting eigenstrain is obtained, we proceed to find the perturbed strains in the fibers. In the absence of residual stress, the perturbed strains in the fibers within the two-phase composite can be derived by using the following equivalence equation consistently:

$$
\mathbf{C}_{0}:\left(\varepsilon^{0}+\varepsilon^{\prime}-\varepsilon^{* *}\right)=\mathbf{C}^{(1)}:\left(\varepsilon^{0}+\varepsilon^{\prime}\right) .
$$

Therefore, by making use of Eqs. (30) and (33), the consistent perturbed strain reads:

$$
\varepsilon^{\prime}=\mathbf{H}: \varepsilon^{0},
$$

where

$$
\mathbf{H}=\left(\Delta \mathbf{C}^{(1)}\right)^{-1} \bullet \mathbf{C}_{0} \bullet\left(\mathbf{A}^{(1)}-\overline{\mathbf{A}}^{(1)}\right)^{-1}-\mathbf{I} .
$$

Since this is not the single-inclusion problem anymore, the interior-point Eshelby tensor is not directly employed to find the consistent perturbed strain.

On the other hand, the perturbed strain can still be approximated with the interior-point Eshelby tensor $\mathbf{S}$ (cf. Eq. (2)). Without resorting to the equivalence equation, one can obtain the following simplified perturbed strain:

$$
\varepsilon^{\prime}=\mathbf{K}: \varepsilon^{0},
$$

where

$$
\mathbf{K}=-\mathbf{S} \bullet\left(\mathbf{A}^{(1)}-\overline{\mathbf{A}}^{(1)}\right)^{-1}
$$



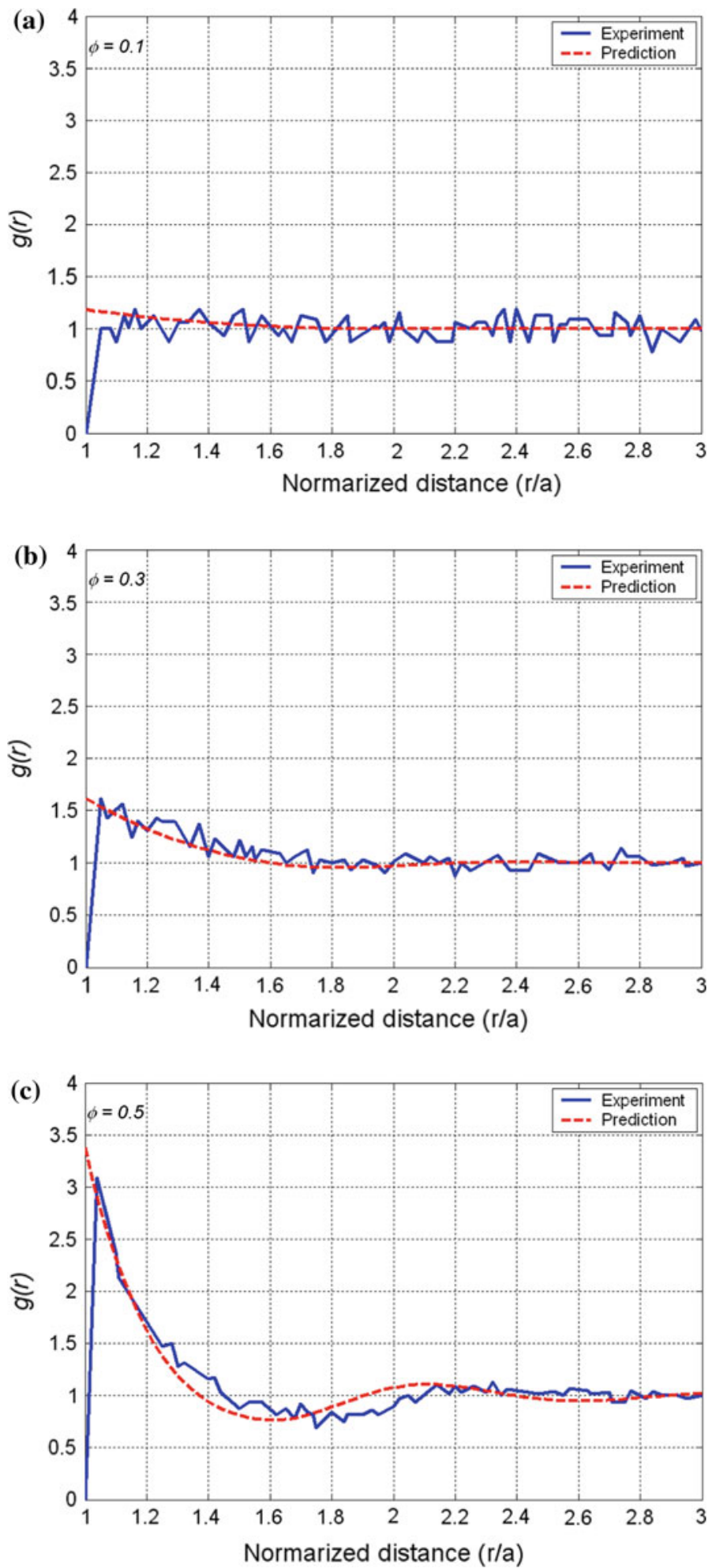

Fig. 3 The comparisons of radial distributions. a The volume fraction $\phi=0.1$, b the volume fraction $\phi=0.3$, $\mathbf{c}$ the volume fraction $\phi=0.5$ 
2.5 Effective elastic stiffness of two-phase fiber composites

Let us consider the volume-averaged strain tensor:

$$
\begin{aligned}
\bar{\varepsilon} & =\varepsilon^{0}+\frac{V_{m}}{V} \overline{\boldsymbol{\varepsilon}}_{m}^{\prime}+\frac{1}{V} \sum_{r=1}^{N} V_{r} \overline{\boldsymbol{\varepsilon}}_{r}^{\prime} \\
& \cong \varepsilon^{0}+\frac{V_{m}}{V}\left[\frac{1}{V_{m}} \sum_{r=1}^{N}\left(\int_{\mathbf{x} \notin \Omega_{r}} \int_{\mathbf{y} \in \Omega_{r}} \mathbf{G}(\mathbf{x}-\mathbf{y}): \overline{\boldsymbol{\varepsilon}}_{r}^{* *}(\mathbf{y}) \mathrm{d} V(\mathbf{y}) \mathrm{d} V(\mathbf{x})\right)\right]+\frac{1}{V} \sum_{r=1}^{N} V_{r} \overline{\boldsymbol{\varepsilon}}_{r}^{\prime} \\
& =\varepsilon^{0}+\frac{1}{V} \sum_{r=1}^{N} V_{r} \bar{\varepsilon}_{r}^{\prime} \\
& =\varepsilon^{0}+\sum_{r=1}^{N} \phi^{(r)} \overline{\boldsymbol{\varepsilon}}^{\prime}{ }_{r} .
\end{aligned}
$$

Here, G represents the Green's function (cf. [5,13]), and volume-averaged perturbed strains are considered. Additionally, the Tanaka-Mori lemma [19] is applied for a cylindrical RVE with a cylindrical fiber to perform the integration. The volume-averaged stress can be expressed as (cf. [7]):

$$
\bar{\sigma}=\mathbf{C}_{0}:\left[\bar{\varepsilon}-\sum_{r=1}^{N} \phi^{(r)} \varepsilon^{r^{* *}}\right] .
$$

Hence, by making use of Eqs. (34), (36), (38) and (39), the effective stiffness of two-phase composites can be rendered as:

$$
\begin{array}{ll}
\mathbf{C}^{*}=\mathbf{C}_{0} \bullet\left[\mathbf{I}+\phi\left(\mathbf{A}^{(1)}-\overline{\mathbf{A}}^{(1)}\right)^{-1} \bullet(\mathbf{I}+\phi \mathbf{H})^{-1}\right], & \text { for consistent formulation, } \\
\mathbf{C}^{*}=\mathbf{C}_{0} \bullet\left[\mathbf{I}+\phi\left(\mathbf{A}^{(1)}-\overline{\mathbf{A}}^{(1)}\right)^{-1} \bullet(\mathbf{I}+\phi \mathbf{K})^{-1}\right], & \text { for simplified formulation. }
\end{array}
$$

We now consider a special case to illustrate the essential feature of the proposed micromechanical formulation. By setting $\overline{\mathbf{A}}=\mathbf{0}$, we can show that

$$
\begin{aligned}
\mathbf{H} & =\left(\Delta \mathbf{C}^{(1)}\right)^{-1} \bullet \mathbf{C}_{0} \bullet\left(\mathbf{A}^{(1)}\right)^{-1}-\mathbf{I} \\
& =\left[\left(\Delta \mathbf{C}^{(1)}\right)^{-1} \cdot \mathbf{C}_{0}+\mathbf{S}\right] \cdot\left[\left(\Delta \mathbf{C}^{(1)}\right)^{-1} \cdot \mathbf{C}_{0}+\mathbf{S}\right]^{-1}-\mathbf{S} \bullet\left[\left(\Delta \mathbf{C}^{(1)}\right)^{-1} \cdot \mathbf{C}_{0}+\mathbf{S}\right]^{-1}-\mathbf{I} \\
& =-\mathbf{S} \bullet\left[\left(\Delta \mathbf{C}^{(1)}\right)^{-1} \bullet \mathbf{C}_{0}+\mathbf{S}\right]^{-1} \\
& =\mathbf{K} .
\end{aligned}
$$

Therefore, in the event of $\overline{\mathbf{A}}=\mathbf{0}$, both Eqs. (40) and (41) reduce to the following equation:

$$
\mathbf{C}^{*}=\mathbf{C}_{0} \bullet\left\{\mathbf{I}+\phi\left[\Delta \mathbf{C}^{(1)} \bullet \mathbf{C}_{0}+(1-\phi) \mathbf{S}\right]^{-1}\right\} .
$$

In essence, Eq. (43) is the effective stiffness predicted by the Mori-Tanaka method [23,24] or the Ju-Chen first-order formulation [7], in which only the far-field interactions are accounted for (cf. [5]). Clearly, the tensor $\overline{\mathbf{A}}$ in the proposed micromechanical formulation is directly linked to the near-field interactions among the fibers. 

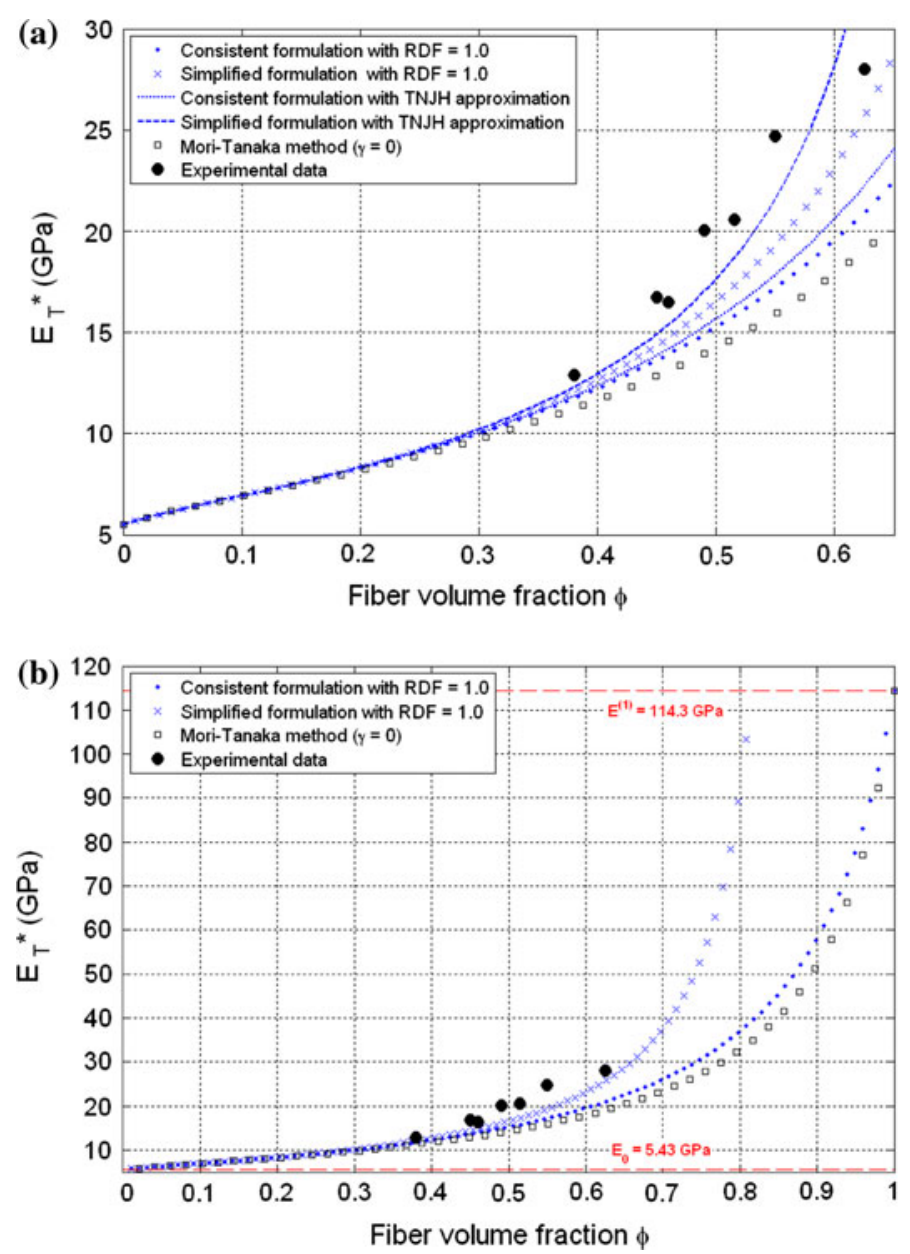

Fig. 4 The comparisons between the theoretical predictions and the experimental data [26] for the effective transverse Young's modulus $E_{T}^{*}$. a The range of volume fraction: $0 \leq \phi \leq 0.65$, $\mathbf{b}$ the range of volume fraction: $0 \leq \phi \leq 1.0$. Glass-epoxy composite: $E_{0}=5.43 \mathrm{GPa}, v_{0}=0.35, E^{(1)}=114.3 \mathrm{GPA}, v^{(1)}=0.22$

\section{Numerical simulations and further discussions}

In this section, a series of micromechanical analytical predictions are presented along with the available experimental data to illustrate the predictive capability of the proposed framework. Here, we focus on the effective elastic moduli of two-phase composites. As shown in Fig. 4a, the numerical simulations with the simplified formulation exhibit better predictions in comparison with the consistent formulation. Further, by introducing the radial distribution function, the theoretical predictions are slightly improved. The prediction with the uniform radial distribution $(g(r)=1)$ may be regarded as the lower bound. In spite of the successful predictive capability of the simplified formulation, it hits a mathematical singularity at around $\phi=0.8$, though such a high volume fraction may not be realistic. By contrast, such a singularity does not exist in the consistent formulation (cf. Fig. 4b). It is noted that micromechanical models can permit $\phi \rightarrow 1.0$ and are particularly amenable to theoretical treatment. Moreover, micromechanical models giving $\phi \rightarrow 1.0$ are appropriate for use in describing practical poly-dispersed suspensions involving a gradation of sizes of inclusions (cf. [25]).

Since the proposed framework is based on the pair-wise interaction solution, it cannot fully account for many-fiber interactions within the composite materials. In addition, we adopt the mean fiber radius for the sake of mathematical simplicity. Hence, the effect due to the size-distribution of fibers is neglected in this study. In addition, the misalignment of fibers may have a considerable effect on the mechanical properties of composites. Because the TNJH approximation [21] is applicable up to $\phi \cong 0.5$, one needs to find a radial distribution function for such a high volume fraction $\phi>0.5$. In practice, once $g(r)$ is involved, the integration 

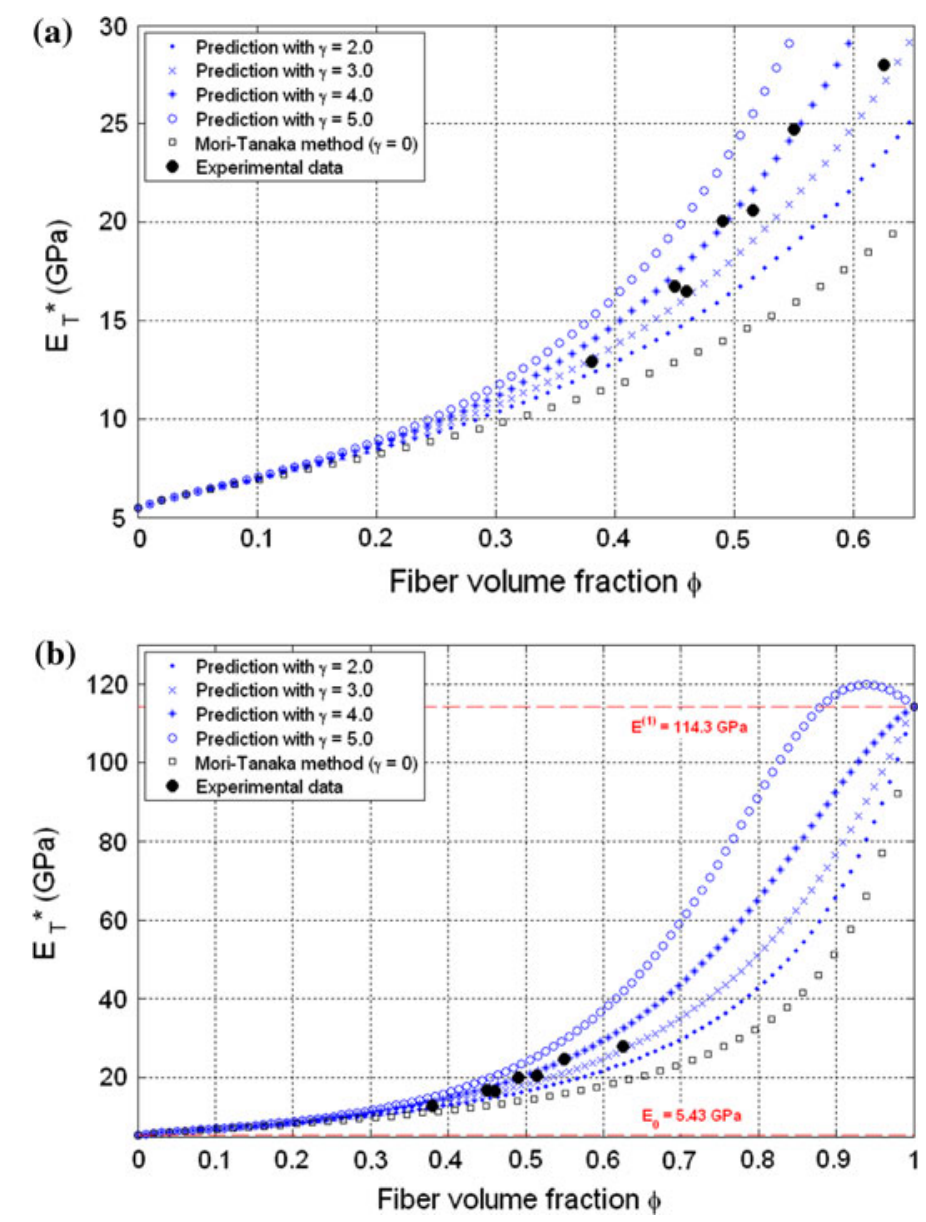

Fig. 5 The comparisons between the theoretical predictions (consistent formulation with various $\gamma$ values) and the experimental data [26] for the effective transverse Young's modulus $E_{T}^{*}$. a The range of volume fraction: $0 \leq \phi \leq 0.65$, $\mathbf{b}$ the range of volume fraction: $0 \leq \phi \leq 1.0$. Glass-epoxy composite: $E_{0}=5.43 \mathrm{GPa}, v_{0}=0.35, E^{(1)}=114.3 \mathrm{GPA}, v^{(1)}=0.22$

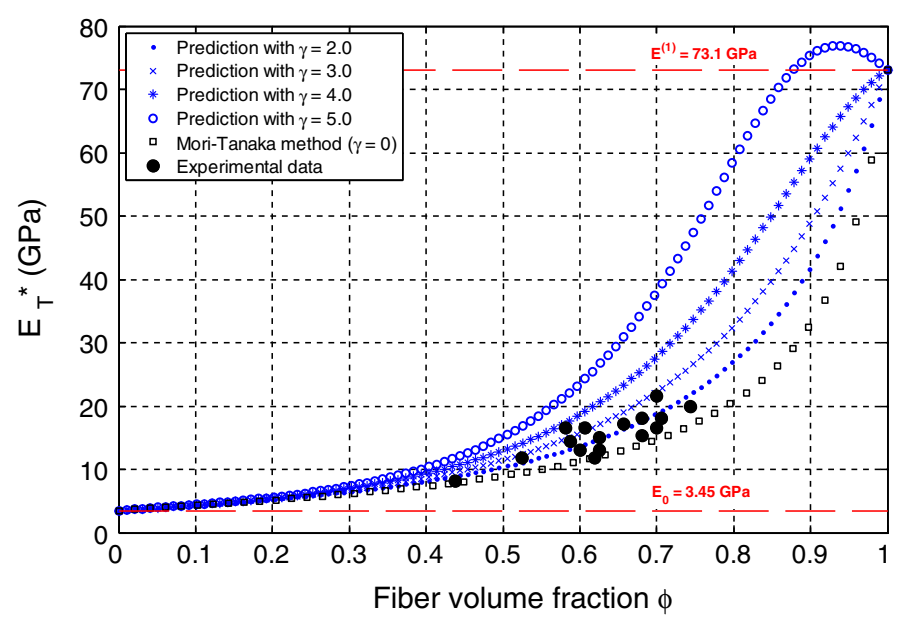

Fig. 6 The comparisons between the theoretical predictions and the experimental data Hahn [27,28] for the effective transverse Young's modulus $E_{T}^{*}$. Glass-epoxy composite: $E_{0}=3.45 \mathrm{GPa}, v_{0}=0.35, E^{(1)}=73.1 \mathrm{GPA}, v^{(1)}=0.22$

of Eq. (31) becomes rather tedious, leading to a significant increase in computation time. Nonetheless, the incorporation of $g(r)$ can slightly improve the micromechanical analytical predictions, particularly for the consistent formulation. To overcome these difficulties, the simplest approach is to approximate Eq. (31) by 
the following expression:

$$
\overline{\mathbf{A}}^{(r)}=\frac{N^{(r)}}{\mathrm{A}} \int_{A \notin A^{(r)}} \hat{\mathbf{G}} \bullet\left(\mathbf{A}^{(r)}\right)^{-1} \bullet \hat{\mathbf{G}} \cdot g(r) d A \approx \frac{N^{(r)}}{A} \gamma \int_{A \notin A^{(r)}} \hat{\mathbf{G}} \bullet\left(\mathbf{A}^{(r)}\right)^{-1} \bullet \hat{\mathbf{G}} d A .
$$

Here, it is assumed that a parameter $\gamma$ signifies the overall effect of $g(r)$ within an RVE. In reality, $\gamma$ is not a constant; it should be at least a function of $\phi$. Due to the approximation, Eq. (44) may not fully reflect the microstructural effects. However, we still consider the effects of probabilistic fiber locations with the approximation in Eq. (44). Therefore, the proposed formulation can be regarded as a mostly-micromechanical formulation. In what follows, to further investigate the proposed micromechanical framework, parametric studies on the parameter $\gamma$ are performed with the consistent formulation. By exercising Eq. (44) with $\gamma=$ constant, we can reproduce various experimental data with good accuracy.

In Fig. 5, the numerical simulations with various values of $\gamma$ are presented. When $2 \leq \gamma \leq 3$, the simulated values are in reasonably good agreement with the experimental data. Despite the simulations being stable without exhibiting the singularity, when $\gamma=5$, the simulation shows a unique behavior; that is, the simulation not only over-predicts the experimental data, but also goes beyond the physical upper bound (i.e., $E^{(1)}=114.3 \mathrm{GPa}$ ). The same phenomenon can be observed in Fig. 6. From these results, it is clear that there

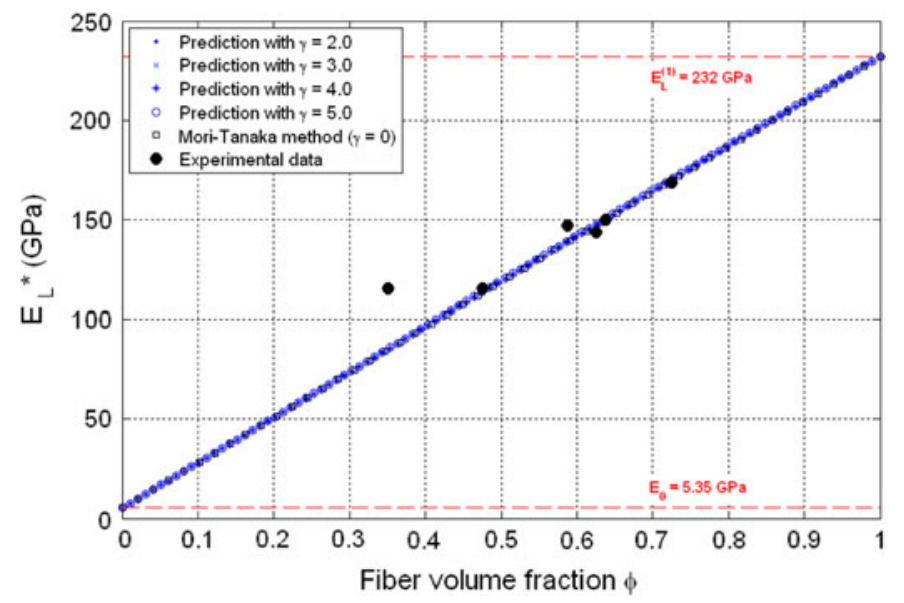

Fig. 7 Comparison between the theoretical predictions and the experimental data $[28,29]$ for the effective longitudinal Young's modulus $E_{L}^{*}$. Carbon-epoxy composite: $E_{0}=5.35 \mathrm{GPa}, v_{0}=0.35, E_{L}^{(1)}=232 \mathrm{GPA}, E_{T}^{(1)}=15 \mathrm{GPa}, v_{L T}^{(1)}=0.22, v_{T T}^{(1)}=0.49$, $\mu_{L T}^{(1)}=24 \mathrm{GPa}$

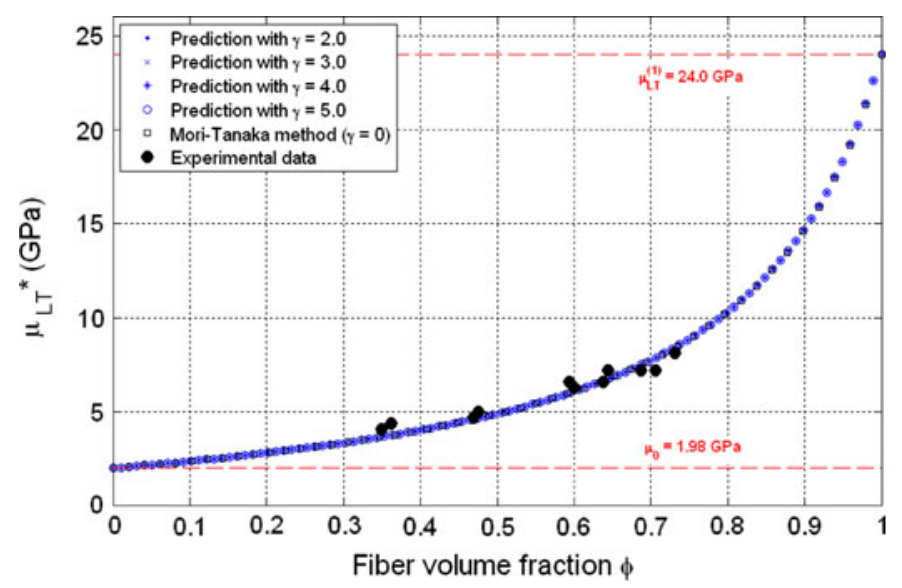

Fig. 8 Comparison between the theoretical predictions and the experimental data $[28,29]$ for the effective transverse shear modulus $\mu_{L T}^{*}$. Carbon-epoxy composite: $E_{0}=5.35 \mathrm{GPa}, v_{0}=0.35, E_{L}^{(1)}=232 \mathrm{GPA}, E_{T}^{(1)}=15 \mathrm{GPa}, v_{L T}^{(1)}=0.22, v_{T T}^{(1)}=0.49$, $\mu_{L T}^{(1)}=24 \mathrm{GPa}$ 
exists an upper bound for $\gamma$, which can reasonably permit simulating physical material properties. Finally, according to our rough estimation, Ju and Zhang's lower and upper bounds [10] correspond to $\gamma \approx 3.0$ and $\gamma \approx 4.0$, respectively.

From Figs. 7, 8, 9, 10 and 11, the comparisons are made for anisotropic fiber reinforced composites. As mentioned in the previous section, the proposed framework is based on the two-dimensional interaction solution (cf. Figs. 1,2). Therefore, the effect of interaction in the longitudinal direction cannot be accommodated. Correspondingly, the effect of $\gamma$ cannot be observed in Figs. 7 and 8. Nonetheless, the predictions can reproduce experimental data with good accuracy. In Figs. 9 and 10, effective transverse properties are compared. Those figures demonstrate the excellent predictive capability of the proposed framework by revealing the minor effect of $\gamma$ upon these composites. This does not imply that interaction is not important. Instead, it exemplifies that a unique combination of the matrix and reinforcing-phase results in unique intensity of near-field interaction. Finally, in Fig. 11, the comparisons are made for the effective Poisson's ratio. For up to $\phi=0.6$, the predictions show moderately good agreement compared with the experimental data. However, for very high fiber volume fractions, relatively large discrepancies exist. Since the $v_{T T}^{*}$ experimental data scatter noticeably for very high volume fractions, it may not be meaningful to compare the theoretical and experimental results.

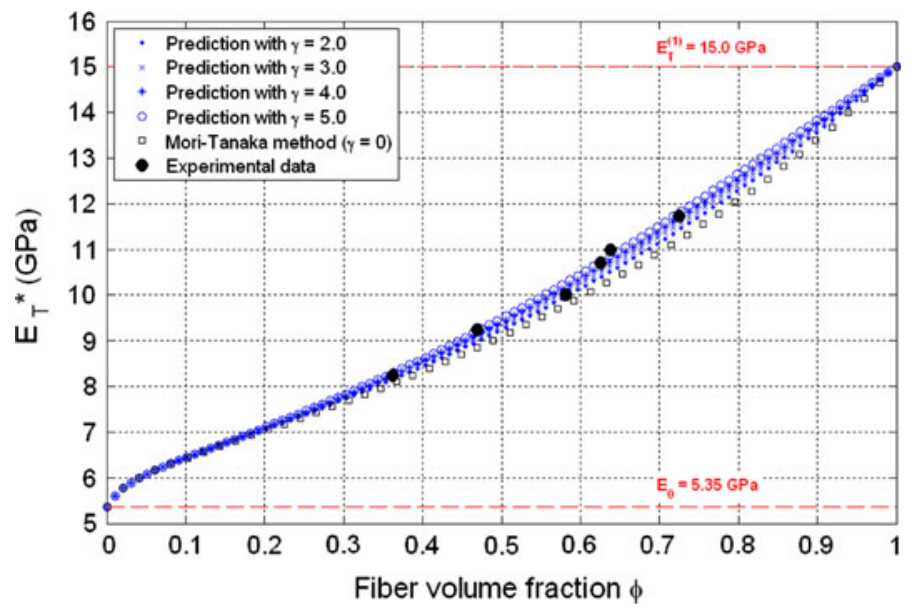

Fig. 9 Comparison between the theoretical predictions and the experimental data $[28,29]$ for the effective transverse Young's modulus $E_{T}^{*}$. Carbon-epoxy composite: $E_{0}=5.35 \mathrm{GPa}, v_{0}=0.35, E_{L}^{(1)}=232 \mathrm{GPA}, E_{T}^{(1)}=15 \mathrm{GPa}, v_{L T}^{(1)}=0.22, v_{T T}^{(1)}=0.49$, $\mu_{L T}^{(1)}=24 \mathrm{GPa}$

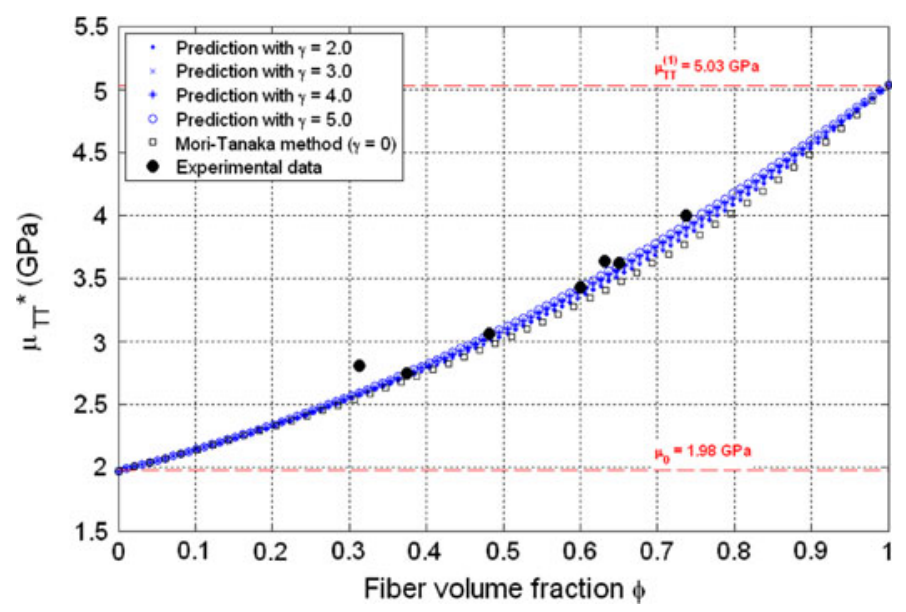

Fig. 10 Comparison between the theoretical predictions and the experimental data $[28,29]$ for the effective shear modulus $\mu_{T T}^{*}$. Carbon-epoxy composite: $E_{0}=5.35 \mathrm{GPa}, v_{0}=0.35, E_{L}^{(1)}=232 \mathrm{GPA}, E_{T}^{(1)}=15 \mathrm{GPa}, v_{L T}^{(1)}=0.22, v_{T T}^{(1)}=0.49$, $\mu_{L T}^{(1)}=24 \mathrm{GPa}$ 


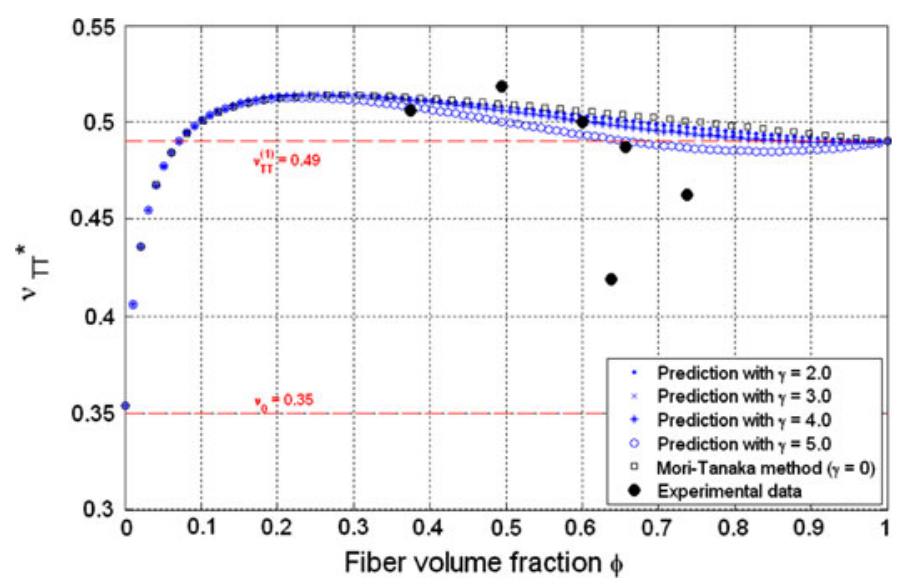

Fig. 11 Comparison between the theoretical predictions and the experimental data [28,29] for the effective Poisson's ratio $v_{T T}^{*}$. Carbon-epoxy composite: $E_{0}=5.35 \mathrm{GPa}, v_{0}=0.35, E_{L}^{(1)}=232 \mathrm{GPA}, E_{T}^{(1)}=15 \mathrm{GPa}, v_{L T}^{(1)}=0.22, v_{T T}^{(1)}=0.49$, $\mu_{L T}^{(1)}=24 \mathrm{GPa}$

\section{Conclusions}

On the foundation of probabilistic pair-wise interaction solution, the higher-order (in $\phi$ ) probabilistic micromechanical formulation is proposed for continuous circular fiber reinforced elastic composites. To accommodate many-fiber interactions and other microstructural effects, the parametric study with the parameter $\gamma$ is conducted. A series of comparisons with available experimental data show that the theoretical predictions can reasonably simulate experimental data when $\gamma \approx 3$. Our investigations for anisotropic-fiber reinforced composites further confirm the predictive capability of the proposed framework. The proposed framework can be extended to accommodate fibers of different sizes or shapes with added effort and complexity.

Acknowledgments This work is in part sponsored by the Faculty Research Grant of the Academic Senate of UCLA (Fund Number 4-592565-19914) and in part by Bellagio Engineering.

Open Access This article is distributed under the terms of the Creative Commons Attribution Noncommercial License which permits any noncommercial use, distribution, and reproduction in any medium, provided the original author(s) and source are credited.

\section{Appendix I}

Let us define the following equation:

$$
\overline{\mathbf{A}}^{(r)}=\frac{N^{(r)}}{\mathrm{A}} \int_{A \notin A^{(r)}} \hat{\mathbf{G}} \bullet\left(\mathbf{A}^{(r)}\right)^{-1} \bullet \hat{\mathbf{G}} \cdot g(r) d A=\frac{N^{(r)}}{\mathrm{A}} \int_{A \notin A^{(r)}} \hat{\mathbf{G}} \bullet \mathbf{L}^{(r)} \bullet \hat{\mathbf{G}} \cdot g(r) d A .
$$

In the case of $g(r)=1$, the components of Eq. (45) can be expressed as

$$
\begin{aligned}
\frac{\bar{A}_{2222}^{(r)}}{\phi^{(r)}}= & L_{2222}^{(r)} \frac{\frac{23699}{10240}-4 v_{0}+2 v_{0}^{2}}{64\left(1-v_{0}\right)^{2}}+L_{2233}^{(r)} \frac{-\frac{3219}{10240}-2 v_{0}+2 v_{0}^{2}}{64\left(1-v_{0}\right)^{2}}+L_{3322}^{(r)} \frac{-\frac{3219}{10240}+2 v_{0}-2 v_{0}^{2}}{64\left(1-v_{0}\right)^{2}} \\
& +L_{3333}^{(r)} \frac{\frac{3219}{10240}-2 v_{0}^{2}}{64\left(1-v_{0}\right)^{2}}+L_{2323}^{(r)} \frac{\frac{8339}{10240}-v_{0}}{16\left(1-v_{0}\right)^{2}}, \\
\frac{\bar{A}_{2233}^{(r)}}{\phi^{(r)}}= & L_{2222}^{(r)} \frac{-\frac{3219}{10240}+2 v_{0}-2 v_{0}^{2}}{64\left(1-v_{0}\right)^{2}}+L_{2233}^{(r)} \frac{-\frac{17261}{10240}+4 v_{0}-2 v_{0}^{2}}{64\left(1-v_{0}\right)^{2}}+L_{3322}^{(r)} \frac{\frac{3219}{10240}+2 v_{0}^{2}}{64\left(1-v_{0}\right)^{2}} \\
& +L_{3333}^{(r)} \frac{-\frac{3219}{10240}-2 v_{0}+2 v_{0}^{2}}{64\left(1-v_{0}\right)^{2}}+L_{2323}^{(r)} \frac{\frac{1901}{10240}-v_{0}}{16\left(1-v_{0}\right)^{2}},
\end{aligned}
$$




$$
\begin{aligned}
\frac{\bar{A}_{3322}^{(r)}}{\phi^{(r)}}= & L_{2222}^{(r)} \frac{-\frac{3219}{10240}-2 v_{0}+2 v_{0}^{2}}{64\left(1-v_{0}\right)^{2}}+L_{2233}^{(r)} \frac{\frac{3219}{10240}+2 v_{0}^{2}}{64\left(1-v_{0}\right)^{2}}+L_{3322}^{(r)} \frac{-\frac{17261}{10240}+4 v_{0}-2 v_{0}^{2}}{64\left(1-v_{0}\right)^{2}} \\
& +L_{3333}^{(r)} \frac{-\frac{3219}{10240}+2 v_{0}-2 v_{0}^{2}}{64\left(1-v_{0}\right)^{2}}+L_{2323}^{(r)} \frac{\frac{1901}{10240}-v_{0}}{16\left(1-v_{0}\right)^{2}}, \\
\frac{\bar{A}_{3333}^{(r)}}{\phi^{(r)}}= & L_{2222}^{(r)} \frac{\frac{3219}{10240}-2 v_{0}^{2}}{64\left(1-v_{0}\right)^{2}}+L_{2233}^{(r)} \frac{-\frac{3219}{10240}+2 v_{0}-2 v_{0}^{2}}{64\left(1-v_{0}\right)^{2}}+L_{3322}^{(r)} \frac{-\frac{3219}{10240}-2 v_{0}+2 v_{0}^{2}}{64\left(1-v_{0}\right)^{2}} \\
& +L_{3333}^{(r)} \frac{\frac{23699}{10240}-4 v_{0}+2 v_{0}^{2}}{64\left(1-v_{0}\right)^{2}}+L_{2323}^{(r)} \frac{\frac{8339}{10240}-v_{0}}{16\left(1-v_{0}\right)^{2}}, \\
\frac{\bar{A}_{2323}^{(r)}=}{\phi^{(r)}}= & L_{2222}^{(r)} \frac{8339-10240 v_{0}}{655360\left(1-v_{0}\right)^{2}}+L_{2233}^{(r)} \frac{1901-10240 v_{0}}{655360\left(1-v_{0}\right)^{2}}+L_{3322}^{(r)} \frac{1901-10240 v_{0}}{655360\left(1-v_{0}\right)^{2}} \\
& +L_{3333}^{(r)} \frac{8339-10240 v_{0}}{655360\left(1-v_{0}\right)^{2}}+L_{2323}^{(r)} \frac{3219}{163840\left(1-v_{0}\right)^{2}},
\end{aligned}
$$

where $v_{0}$ is Poisson's ratio of the matrix material.

\section{Appendix II}

In the TNJH approximation [21], the RDF takes the form:

$$
g(r)= \begin{cases}g^{\operatorname{dep}}(r), & \text { for } 2 a \leq r \leq r^{*}, \\ g^{\operatorname{str}}(r), & \text { for } r^{*} \leq r \leq \infty,\end{cases}
$$

where the "depletion" (d) and "structural" (s) parts have the following form:

$$
\begin{aligned}
g^{\operatorname{dep}}(r) & =\frac{A}{r} e^{\mu(r-2 a)}+\frac{B}{r} \cos (\beta[r-2 a]+\gamma) \cdot e^{\alpha(r-2 a)}, \\
g^{\mathrm{str}}(r) & =1+\frac{C}{r} \cos (\omega r+\delta) \cdot e^{-\kappa r} .
\end{aligned}
$$

It is noted that $r^{*}$ is the position for the first minimum of $g(r)$, and it reads

$$
r^{*}=2 a\left(2.0116-1.0647 \phi+0.0538 \phi^{2}\right) .
$$

Moreover, the following equations need to be satisfied at $r=r^{*}$ :

$$
\begin{aligned}
g^{\mathrm{dep}}\left(r^{*}\right) & =g^{\mathrm{str}}\left(r^{*}\right), \\
\left.\frac{d}{\mathrm{~d} r} g^{\mathrm{dep}}(r)\right|_{r=r^{*}} & =\left.\frac{d}{\mathrm{~d} r} g^{\operatorname{str}}(r)\right|_{r=r^{*}} .
\end{aligned}
$$

The coefficients in Eqs. (52) and (53) are rendered as

$$
\begin{aligned}
A & =2 a \cdot g_{\sigma}^{\text {expt }}-B \cos \gamma, \\
B & =\frac{g_{m}-\left(2 a \cdot g_{\sigma}^{\text {expt }} / r^{*}\right) \cdot e^{\mu\left(r^{*}-2 a\right)}}{\cos \left(\beta\left[r^{*}-2 a\right]+\gamma\right) \cdot e^{\alpha\left(r^{*}-2 a\right)}-\cos \gamma \cdot e^{\mu\left(r^{*}-2 a\right)}} r^{*}, \\
C & =\frac{r^{*}\left(g_{m}-1\right) \cdot e^{\kappa r^{*}}}{\cos \left(\omega r^{*}+\delta\right)}, \\
\delta & =-\omega r^{*}-\arctan \frac{\kappa r^{*}+1}{\omega r^{*}},
\end{aligned}
$$


where

$$
\begin{aligned}
g_{m} & =1.0286-0.6095 \phi+3.7581 \phi^{2}-21.3651 \phi^{3}+42.6344 \phi^{4}-33.8485 \phi^{5}, \\
g_{\sigma}^{e x p t} & =\frac{1}{4 \phi}\left(\frac{1+\phi+\phi^{2}-2 / 3 \phi^{3}-2 / 3 \phi^{4}}{(1-\phi)^{4}}-1\right) \\
\alpha & =\frac{1}{2 a}\left(44.554+79.868 \phi+116.432 \phi^{2}-44.652 \cdot e^{(2 \phi)}\right) \\
\beta & =\frac{1}{2 a}\left(-5.022+5.857 \phi+5.089 \cdot e^{(-4 \phi)}\right) \\
\omega & =\frac{1}{2 a}\left(-0.682 e^{(-24.697 \phi)}+4.720+4.450 \phi\right) \\
\kappa & =\frac{1}{2 a}\left(4.674 \cdot e^{(-3.935 \phi)}+3.536 \cdot e^{(-56.270 \phi)}\right) \\
\mu & =\frac{\phi}{2 a(1-\phi)}\left(-1+\frac{d}{2 \phi}+\frac{\phi}{d}\right) \\
d & =\left\{2 \phi\left[\phi^{2}-3 \phi-3+\sqrt{3\left(\phi^{4}-2 \phi^{3}+\phi^{2}+6 \phi+3\right)}\right]\right\}^{1 / 3} .
\end{aligned}
$$

Finally, on the basis of Eq. (56), the unknown coefficient $\gamma$ can be found by solving the following equation:

$$
f_{1}+f_{2}=0
$$

where

$$
\begin{aligned}
& f_{1}=\frac{A \cdot e^{\mu\left(r^{*}-2 a\right)}}{\left(r^{*}\right)^{2}} \cdot\left(\mu \cdot r^{*}-1\right) \\
& f_{2}=\frac{B}{\left(r^{*}\right)^{2}}\left\{\cos \left(\beta\left[r^{*}-2 a\right]+\gamma\right) \cdot\left(\alpha \cdot r^{*}-1\right)-\beta \cdot r^{*} \sin \left(\beta\left[r^{*}-2 a\right]+\gamma\right)\right\}
\end{aligned}
$$

\section{References}

1. Budiansky, B., Cui, Y.L.: Toughening of ceramics by short aligned fibers. Mech. Mater. 21, 139-146 (1995)

2. Agarwal, B.D., Broutman, L.J. Chandrashekhara, K.C.: Analysis and performance of fiber reinforced composites. 3rd edn. Wiley, New York (2006)

3. Whitney, J.M.: Elastic moduli of unidirectional composites with anisotropic filaments. J. Comps. Mater. 1, 188-193 (1967)

4. Eshelby, J.D.: The determination of the elastic field of an ellipsoidal inclusion, and related problems. Proc. R. Soc. Lond. A 241, 376-396 (1957)

5. Qu, J., Cherkaoui, M.: Fundamentals of micromechanics of solids. Wiley, New York (2006)

6. Moschovidis, Z.A., Mura, T.: Two-ellipsoidal inhomogeneities by the equivalent inclusion method. ASME J. Appl. Mech. 42, 847-852 (1975)

7. Ju, J.W., Chen, T.M.: Micromechanics and effective moduli of elastic composites containing randomly dispersed ellipsoidal inhomogeneities. Acta Mech. 103, 103-121 (1994)

8. Ju, J.W., Chen, T.M.: Effective elastic moduli of two-phase composites containing randomly dispersed spherical inhomogeneities. Acta Mech. 103, 123-144 (1994)

9. Ju, J.W., Chen, T.M.: Micromechanics and effective elastoplastic behavior of two-phase metal matrix composites. ASME J. Eng. Mater. Tech. 116, 310-318 (1994)

10. Ju, J.W., Zhang, X.D.: Micromechanics and effective transverse elastic moduli of composites with randomly located aligned circular fibers. Int. J. Solids Struct. 35, 941-960 (1998)

11. Ju, J.W., Zhang, X.D.: Effective elastoplastic behavior of ductile matrix composites containing randomly located aligned circular fibers. Int. J. Solids Struct. 38, 4045-4069 (2001)

12. Lin, P.J., Ju, J.W.: Effective elastic moduli of three-phase composites with randomly located and interacting spherical particles of distinct properties. Acta Mech. 208, 11-26 (2009)

13. Mura, T.: Micromechanics of defects in solids. 2nd edn. Martinus Nijhoff Publishers, Dordrecht (1987)

14. Nemat-Nasser, S., Hori, M.: Micromechanics: overall properties heterogeneous materials. Elsevier, The Netherlands (1993)

15. Hu, G.K., Weng, G.J.: Influence of thermal residual stress on the composite macroscopic behavior. Mech. Mater. 27, 229-240 (1998)

16. Liu, H.T., Sun, L.Z.: Effects of thermal residual stress on effective elastoplastic behavior of metal matrix composites. Int. J. Solids Struct. 41, 2189-2203 (2004) 
17. Ju, J.W., Yanase, K.: Elastoplastic damage micromechanics for elliptical fiber composites with progressive partial fiber debonding and thermal residual stresses. Theoret. Appl. Mech. 35, 137-170 (2008)

18. Ju, J.W., Yanase, K.: Micromechanics and effective elastic moduli of particle-reinforced composites with near-field particle interactions. Acta Mech. doi:10.1007/s00707-010-0337-2 (2010)

19. Tanaka, K., Mori, T.: Note on volume integral of the elastic field around an ellipsoid inclusion. J. Elast. 2, 199-200 (1972)

20. Li, S., Wang, G.: Introduction to micromechanics and nanomechanics. World Scientific Publishing Co. Pte. Ltd., Singapore (2008)

21. Trokhymchuk, A., Nezbeda, I., Jirsák, J., Henderson, D.: Hard-sphere radial distribution function again. J. Chem. Phys. 123, 024501-1-024501-10 (2005)

22. Everett, R.K.: Quantification of random fiber arrangements using a radial distribution function approach. J. Comps. Mater. 30, 748-758 (1996)

23. Mori, T., Tanaka, K.: Average stress in matrix and average elastic energy of materials with misfitting inclusions. Acta Metall. 21, 571-574 (1973)

24. Zhao, Y.H., Tandom, G.P., Weng, G.J.: Elastic moduli for a class of porous materials. Acta Mech. 76, 105-131 (1989)

25. Christensen, R.M.: A critical evaluation for a class of micromechanics models. J. Mech. Phys. Solids 38, 379-404 (1990)

26. Uemura, M., et al. : On the stiffness of filament wound materials (in Japanese). Rep. Inst. Space Aeronaut. Sci. 4, 448-463 (1968)

27. Tsai, S.W., Hahn, H.T.: Introduction to composite materials. Technomic Publishing, Lancaster (1980)

28. Huang, Z.M.: Micromechanical prediction of ultimate strength of transversely isotropic fibrous composites. Int. J. Solids Struct. 38, 4147-4172 (2001)

29. Kriz, R.D., Stinchcomb, W.W.: Elastic moduli of transversely isotropic graphite fibers and their composites. Exp. Mech. 19, 41-49 (1979) 